\title{
Lidamycin induces marked G2 cell cycle arrest in human colon carcinoma HT-29 cells through activation of p38 MAPK pathway
}

\author{
XIA LIU, CHUNJING BIAN, KAIHUAN REN, HAIXIA JIN, BAOWEI LI and RONG-GUANG SHAO \\ Institute of Medicinal Biotechnology, Peking Union Medical College, \\ Chinese Academy of Medical Sciences, Beijing 100050, P.R. China
}

Received October 12, 2006; Accepted November 16, 2006

\begin{abstract}
Lidamycin (LDM), a member of the enediyne antibiotic family, is presently undergoing phase I clinical trials in P.R. China. In this study, we investigated the mechanisms of LDM-induced cell cycle arrest in order to support its use in clinical cancer therapy. Using human colon carcinoma HT-29 cells, we observed that LDM induced G2 cell cycle arrest in a time- and dose-dependent manner. LDM-induced G2 arrest was associated with increasing phosphorylation of Chk1, Chk2, Cdc25C, Cdc2 and expression of Cdc2 and cyclin B1. In addition, cytoplasmic localization of cyclin B1 was also involved in LDM-induced G2 arrest. Moreover, we found that p38 MAPK pathway contributed to LDM-induced G2 arrest. Inhibition of p38 MAPK by its inhibitor SB203580 not only attenuated LDM-induced G2 arrest but also potentiated LDMinduced apoptosis, which was accompanied by decreasing phosphorylation of $\mathrm{Cdc} 2$ and increasing expression of FasL and phosphorylation of JNK. Finally, we demonstrated that cells at G1 phase were more sensitive to LDM. Together, our findings suggest that $\mathrm{p} 38$ MAPK signaling pathway is involved in LDM-induced G2 arrest, at least partly, and a combination of LDM with p38 MAPK inhibitor may represent a new strategy for human colon cancer therapy.
\end{abstract}

\section{Introduction}

Cell cycle arrest and apoptosis are common phenomena after DNA-damaging treatment (1-3). In response to DNA damage, ATM and ATR are activated, and then ATM phosphorylates

Correspondence to: Professor Rong-guang Shao, Institute of Medicinal Biotechnology, Chinese Academy of Medical Sciences, 1 Tiantan Xili, Beijing 100050, P.R. China

E-mail: shaor@bbn.cn

Abbreviations: LDM, lidamycin; PI, propidium iodide; MAPK, mitogen-activated protein kinase; JNK, c-Jun N-terminal kinase; ERK, extracellular signal regulated kinase; DMSO, dimethyl sulfoxide; ATM, ataxia-telangiectasia-mutated; ATR, ATM- and Rad-3-related protein kinase; FITC, fluorescein isothiocyanate; PARP, poly(ADP-ribose) polymerase

Key words: lidamycin, G2 arrest, MAPK, HT-29 cells
Chk2 on Thr-68 leading to Chk2 kinase activation (4), while both ATM and ATR phosphorylate Chk1 on Ser-317 and Ser-345 resulting in its activation $(5,6)$. Activated Chk1 and Chk2 phosphorylate Cdc25C on Ser-216, which promotes the interaction of $\mathrm{Cdc} 25 \mathrm{C}$ with $14-3-3$ proteins and inhibits the ability of $\mathrm{Cdc} 25 \mathrm{C}$ to activate $\mathrm{Cdc} 2 /$ cyclin $\mathrm{B} 1$, resulting in $\mathrm{G} 2$ cell cycle arrest (7). In addition, several lines of evidence demonstrated that constitutive nuclear accumulation of cyclin $\mathrm{B} 1$ caused a reduction in damage-induced $\mathrm{G} 2 / \mathrm{M}$ arrest, thereby raising the possibility that DNA-damage signaling acts in part by stabilizing the cytoplasmic localization of cyclin B1 $(8,9)$.

Recently, it has been demonstrated that other pathways contribute to the $\mathrm{G} 2 / \mathrm{M}$ checkpoint, among which include MAPK cascades. In mammals, MAPKs are divided into three major groups, p38 MAPK, JNK and ERK. Since Bulavin et al found that $\mathrm{p} 38$ MAPK was required for ultraviolet radiation induced G2/M arrest (10), the role of p38 MAPK in G2/M cell cycle checkpoint has been confirmed by other studies $(11,12)$. Accumulating evidence also indicates that ERK has been implicated in $\mathrm{G} 2 / \mathrm{M}$ checkpoint $(13,14)$. In contrast, several studies have demonstrated that inhibition of JNK can induce G2/M arrest $(15,16)$. Furthermore, recent data suggest that MAPKs may mediate apoptotic signaling induced by anticancer drugs. However, these MAPKs have been shown to exert both antiapoptotic and proapoptotic effects in different studies. For example, p38 MAPK activation is a critical pathway in glucocorticoid-induced cell death (17), whereas inhibition of p38 can potentiate sulindac sulfide induced apoptosis in colon carcinoma cells (18). Together, these results indicate that the relationship of MAPKs with cell cycle arrest and apoptosis is complicated and may be dependent on the cellular context, cell type and stimuli.

Lidamycin (LDM, originally named C-1027) is a member of the enediyne antibiotic family, which was isolated from a Streptomyces globisporus C1027 strain (19). As a DNAdamaging agent, LDM is characterized by inducing a higher ratio of DNA double-strand breaks (DSBs) than single-strand breaks (SSBs) (20). It is well known that DSB is the most severe DNA lesion, which may explain the highly potent cytotoxicity of LDM towards cancer cells. It has been reported that LDM can induce cell cycle arrest (21), and LDM-induced cell cycle arrest was dependent on drug concentration and p53 status (22). However, the detailed molecular mechanisms and signaling pathways of LDM-induced cell cycle arrest are not well understood. 
In the present study, we intended to further elucidate the molecular mechanisms of LDM-induced cell cycle arrest in HT-29 cells. Specifically, we examined the influence of p38 MAPK signaling upon LDM-induced G2 arrest and cell death. Finally, we investigated whether the sensitivity of HT-29 cells to LDM was cell cycle dependent.

\section{Materials and methods}

Cell culture and chemicals. Human colon carcinoma HT-29 cells were cultured in DMEM (Gibco BRL, Grand Island, NY) supplemented with $10 \%$ heat-inactivated fetal bovine serum (Sigma Chemical Co., St. Louis, Missouri), 100 U/ml penicillin, and $100 \mu \mathrm{g} / \mathrm{ml}$ streptomycin at $37^{\circ} \mathrm{C}$ in a humidified atmosphere containing $5 \% \mathrm{CO}_{2}$.

LDM was generously provided by Professor Jin (Institute of Medicinal Biotechnology, CAMS). LDM stock solution $(10 \mu \mathrm{M})$ was prepared in water and stored at $-70^{\circ} \mathrm{C}$. SB203580 was purchased from Calbiochem (San Diego, CA) and dissolved in DMSO (Sigma). All other chemicals were purchased from Sigma.

Cell synchronization. HT-29 cells were synchronized as described previously with a little modification (23). Briefly, the cells were synchronized at the $\mathrm{G} 1$ phase by placing unsynchronized cells in the serum-free medium for $48 \mathrm{~h}$ and then replacing medium containing $10 \%$ serum for $3 \mathrm{~h}$. To obtain cells synchronized at the $\mathrm{S}$ phase, the cells were treated with $5 \mu \mathrm{g} / \mathrm{ml}$ aphidicolin for $24 \mathrm{~h}$, and then washed and maintained in the drug-free medium for $3 \mathrm{~h}$, while maintaining cells in the drug-free medium for $9 \mathrm{~h}$ to obtain the G2/M phase cells. Mitotic cells were synchronized by incubating cells with $0.4 \mu \mathrm{g} / \mathrm{ml}$ nocodazole for $24 \mathrm{~h}$.

Drug treatment. For all treatment of LDM, cells were exposed to LDM as described below in each experiment. For treatment of SB203580, cells were pretreated with $20-\mu \mathrm{M}$ SB203580 for $1 \mathrm{~h}$, after which cells were exposed to LDM for $48 \mathrm{~h}$ in the presence of SB203580. The final DMSO concentration did not exceed $0.1 \%$ ( $\mathrm{vol} / \mathrm{vol})$.

Antibodies. Cyclin B1 (GNS1), cyclin A (H-432), Cdc2 (C-19), p21 (F-5), actin (I-19), rabbit p38 MAPK (C-20), JNK1 (C-17) and PARP (F-2) antibodies were from Santa Cruz Biotechnology (Santa Cruz, CA). Chk1 (2345), Chk2 (2662), phospho-Cdc2 (Tyr-15) (9111), phospho-Chk1 (Ser-345) (2341), phospho-Chk2 (Thr-68) (2661), phospho-Cdc25C (Ser 216) (9528), phospho-p38 MAP kinase (Thr180/Tyr182) (9211), phospho-SAPK/JNK MAP kinase (Thr-183/Tyr-185) (9251) and phospho-ATF2 (Thr-71) (9221) antibodies were from Cell Signaling Technology (Beverly, MA). FasL (Ab-1) antibody was purchased from Calbiochem.

Flow cytometry analysis. Floating and attached cells were collected. Cells were fixed in ice-cold $70 \%$ ethanol and stored at $-20^{\circ} \mathrm{C}$. Samples were then washed twice in PBS and resuspended in a solution of PI $(50 \mu \mathrm{g} / \mathrm{ml})$ and RNase A $(0.5 \mathrm{mg} / \mathrm{ml})$ in PBS for $30 \mathrm{~min}$ at $37^{\circ} \mathrm{C}$ in the dark. The stained cells were filtered through $40 \mu \mathrm{m}$ gauze, and the single-cell suspensions were analyzed on an Epics-XL flow cytometer (Coulter Electronics, Inc., Miami, FL) using Mcycle software.

In MPM-2/PI bivariate flow cytometry, cells were labeled with MPM-2 antibody (final concentration of $1 \mu \mathrm{g}$ of MPM-2 antibody/ml) for $1 \mathrm{~h}$ at $4^{\circ} \mathrm{C}$. Cells were washed once with PBS and incubated with antimouse IgG conjugated with FITC antibody (Santa Cruz Biotechnology) for $1 \mathrm{~h}$ at room temperature in the dark. After washing with PBS, cells were resuspended in $5 \mu \mathrm{g} / \mathrm{ml}$ PI containing $50 \mu \mathrm{g} / \mathrm{ml}$ RNase A for $30 \mathrm{~min}$ in the dark (24). Samples were analyzed on an EpicsXL flow cytometer. The MPM-2 positive cells (mitotic cells) showed increased green fluorescence, thus shifting above the baseline of the dot plot.

Western blotting. The procedure has been described previously (22). Briefly, cells were harvested, and washed with PBS solution. The whole cellular extracts were prepared by incubating cells on ice in lysis buffer. The cell lysates were cleared by centrifugation at $12,000 \times \mathrm{g}$ for $12 \mathrm{~min}$. Protein concentrations were determined by Bradford assay. Equal amounts of lysate $(40 \mu \mathrm{g})$ were resolved by SDS-PAGE and transferred to polyvinylidene difluoride membrane (Millipore Corp., Bedford, MA). Membranes were blocked in TBST containing 5\% nonfat skim milk at room temperature for $2 \mathrm{~h}$ and probed with primary antibodies overnight at $4{ }^{\circ} \mathrm{C}$. Then membranes were blotted with an appropriate horseradish peroxidase-linked secondary antibody (Santa Cruz Biotechnology). Proteins were visualized using enhanced chemiluminescence Western blotting detection reagents (Amersham Pharmacia Biotech, Inc., Piscataway, NJ).

Indirect immunofluorescence. Cells were grown on sterile coverslips and treated with drugs for an indicated time. Then cells were fixed with $3.7 \%$ formaldehyde in PBS for $10 \mathrm{~min}$ at $37^{\circ} \mathrm{C}$ and permeabilized in $0.2 \%$ Triton-100 in PBS for $10 \mathrm{~min}$ at room temperature. Nonspecific binding sites were blocked by incubating the cells with $3 \%$ bovine serum albumin in PBS for $1 \mathrm{~h}$ at $37^{\circ} \mathrm{C}$, and stained with mouse monoclonal anti-cyclin $\mathrm{B} 1$ antibody overnight at $4^{\circ} \mathrm{C}$ as described previously (8). After washing 3 times with PBS, cells were stained with FITC-labelled anti-rabbit IgG antibody for $1 \mathrm{~h}$ at room temperature in the dark. After incubation, the coverslips were washed with PBS, and imaged using an Olympus IX-70 inverted fluorescent microscope (Olympus, Tokyo, Japan).

Hoechst 33342 staining. Cells were treated with LDM or cotreated with LDM and SB203580 for $48 \mathrm{~h}$, and then the floating and attached cells were collected. After being centrifuged and washed with PBS, cells were fixed with $4 \%$ paraformaldehyde for $10 \mathrm{~min}$ at room temperature and then washed once with PBS. Hoechst $33342(5 \mu \mathrm{g} / \mathrm{ml})$ was added to the fixed cells, incubated for $10 \mathrm{~min}$ at room temperature. Then cells were spread on coverslips, and imaged using an Olympus IX-70 inverted fluorescent microscope. For each determination, at least 300 cells were counted from more than 5 random microscopic fields. The percentage of apoptotic death cells was calculated from the number of cells with apoptotic (condensed and fragmented nuclei) nuclear morphology divided by the total number of cells examined. 
A

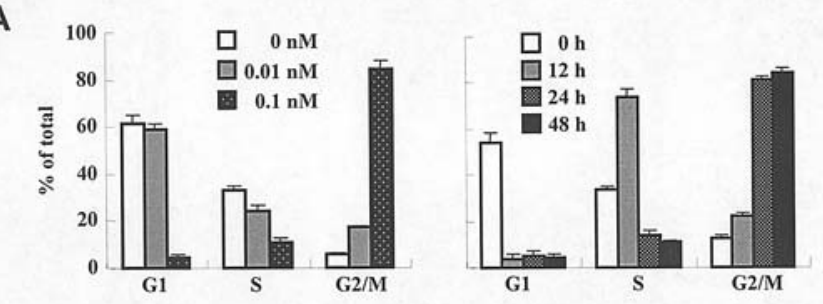

B
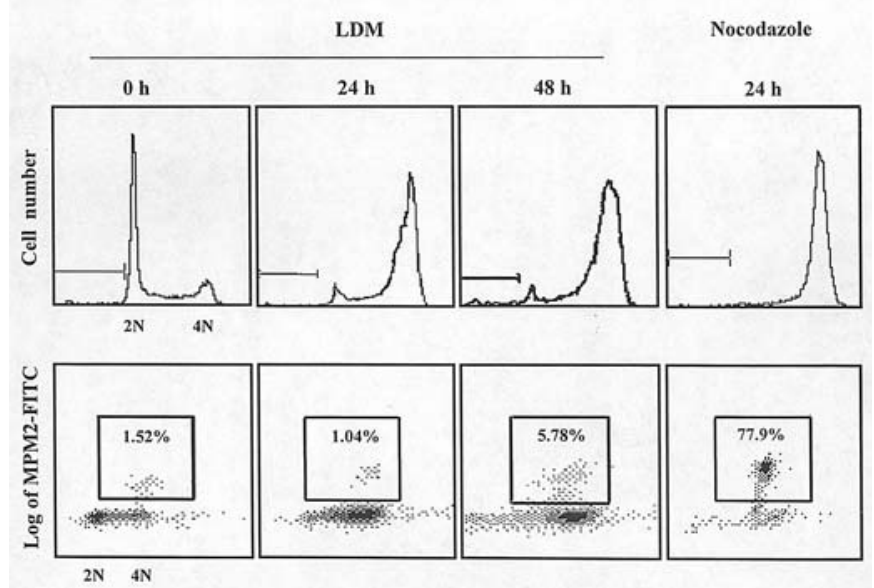

Figure 1. LDM induces G2 phase arrest in HT-29 cells. (A) PI staining for cell cycle distribution. Cells were treated with LDM for $48 \mathrm{~h}$ at the indicated concentrations (left panel) or treated with $0.1 \mathrm{nM}$ LDM for the indicated time (right panel), and then the cell cycle distribution was determined by flow cytometry after PI staining. Error bars represent means \pm SD. (B) The MPM-2/PI bivariate flow cytometry for the MPM-2 expression. Cells were treated with $0.1 \mathrm{nM} \mathrm{LDM}$ at the indicated times as described in 'Materials and methods'. Cells treated with $0.4 \mu \mathrm{g} / \mathrm{ml}$ nocodazole for $24 \mathrm{~h}$ served as a positive control. Mitotic cells are indicated by a box in the dot plots. All data are representative of three independent experiments.

\section{Results}

LDM induces G2 arrest in HT-29 cells. First, we determined the cell cycle alteration after LDM treatment in HT-29 cells. Cells were labeled with PI and analyzed by DNA flow cytometry. After treatment with LDM, the cells significantly accumulated in the $\mathrm{G} 2 / \mathrm{M}$ phase in both a time- and concentration-dependent manner (Fig. 1A). At $1 \mathrm{nM}$, the apoptotic cells were markedly increased, as detected in a prominent sub-G1 peak (data not shown). For this study, we chose $0.1 \mathrm{nM}$ for the subsequent assays before cells underwent apoptosis.

Conventional DNA flow cytometry does not distinguish cells in the G2 phase from cells in the M phase, because cells in both phases have the same DNA content (25). Therefore, we used an MPM-2 antibody that reacted specifically with phosphoprotein presented in $\mathrm{M}$ phase cells in combination with PI staining to further analyze the effects of LDM on cell cycle progression (26). After cells were treated with $0.4 \mu \mathrm{g} /$ $\mathrm{ml}$ nocodazole (a known agent inducing $\mathrm{M}$ arrest) for $24 \mathrm{~h}$, $77.9 \%$ of cells were MPM-2 positive (mitotic cells), indicating that most cells were in $\mathrm{M}$ phase which validated the method (Fig. 1B). In contrast, although the result of PI staining indicated that $>80 \%$ of cells were accumulated in

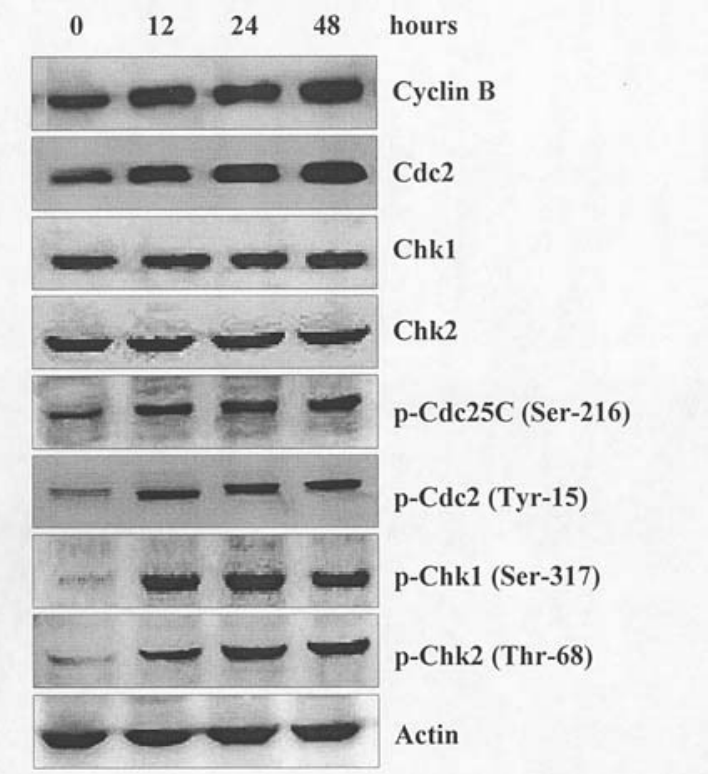

Figure 2. Changes of cell cycle related proteins in LDM-treated HT-29 cells Cells were treated with $0.1 \mathrm{nM}$ LDM for the indicated time. Cell lysates were prepared for SDS-PAGE and analyzed by Western blotting as described in 'Materials and methods'. Actin was used as a loading control. Data are representative of three independent experiments.

G2/M phase when treated with $0.1 \mathrm{nM} \mathrm{LDM}$ for $24 \mathrm{~h}$ (Fig. 1A), only $1.04 \%$ of cells were MPM-2 positive (Fig. 1B), indicating that most cells were arrested in G2 phase. Although the mitotic cells were slightly increased at $48 \mathrm{~h}$ (from $1.04 \%$ to $5.78 \%$ ), most of the cells were MPM-2 negative, suggesting cells were still mainly accumulated in G2 phase.

Changes of cell cycle related proteins by LDM. Based on the above results, we next investigated the effect of LDM on G2 phase-associated regulatory molecules. The data showed that LDM treatment resulted in an increase in the protein expression of cyclin B1 and Cdc2 (Fig. 2). In addition, the levels of phosphorylated Cdc2 (Tyr-15) and phosphorylated Cdc25C (Ser-216) were increased by LDM (Fig. 2). Although total protein levels of Chk1 and Chk2 were unchanged, activated forms of Chk1 (phosphorylated at Ser-317) and Chk2 (phosphorylated at Thr-68) were obviously increased after LDM treatment (Fig. 2), indicating that both Chk1 and Chk2 pathways were activated by LDM.

It has been reported that $\mathrm{p} 21$ plays a role in inducing the G2 arrest and expression of p21 can be induced in a p53independent pathway $(27,28)$. Thus, we investigated whether LDM can induce p21 expression in p53 mutant HT-29 cells. After LDM treatment, the protein levels of p21 were undetected in both control and treated HT-29 cells (data not shown), indicating that LDM-induced G2 arrest was independent of p21 and LDM cannot induce p21 expression in p53 mutant HT-29 cells.

Cytoplasmic localization of cyclin B1 in LDM-treated cells. Since cytoplasmic localization of cyclin B1 has been implicated in establishing G2 arrest after DNA damage $(8,9)$, we examined the subcellular localization of cyclin B1. We found that cyclin B1 mostly accumulated in the cytoplasm after LDM 


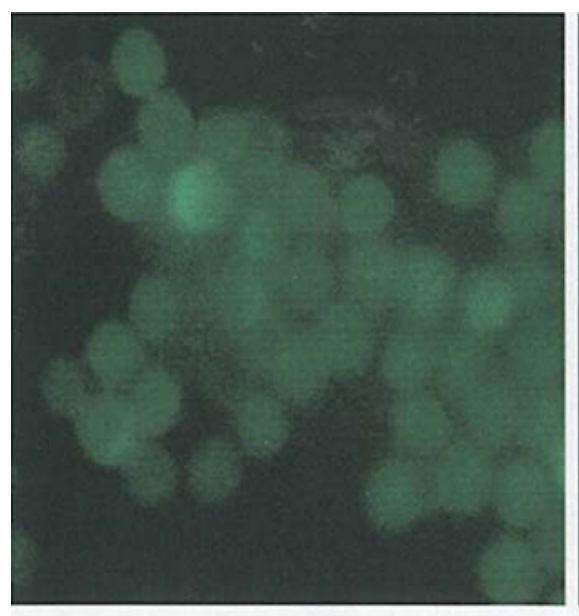

M phase

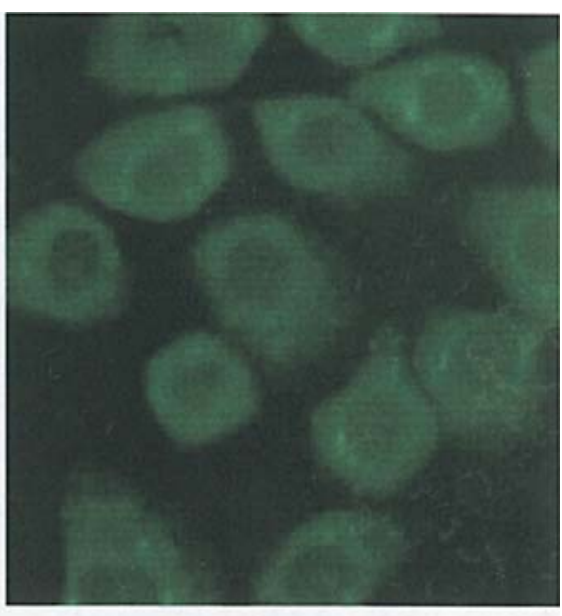

LDM treatment

Figure 3. Cytoplasmic localization of cyclin B1 after LDM treatment. HT-29 cells were treated with $0.1 \mathrm{nM}$ LDM for $24 \mathrm{~h}$, and then fixed and stained as described in 'Materials and methods'. Mitosis phase synchronized cells were used as control. Green fluorescence indicates the localization of cyclin B1. Data are representative of two independent experiments.

A

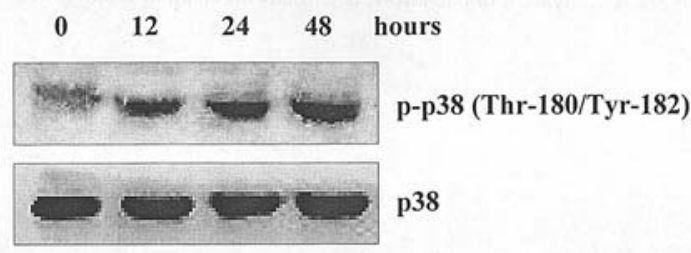

B

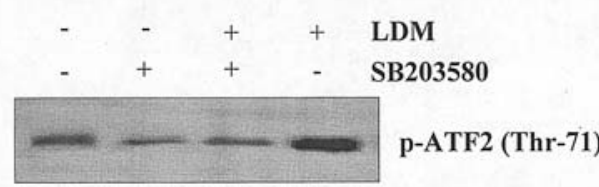

C

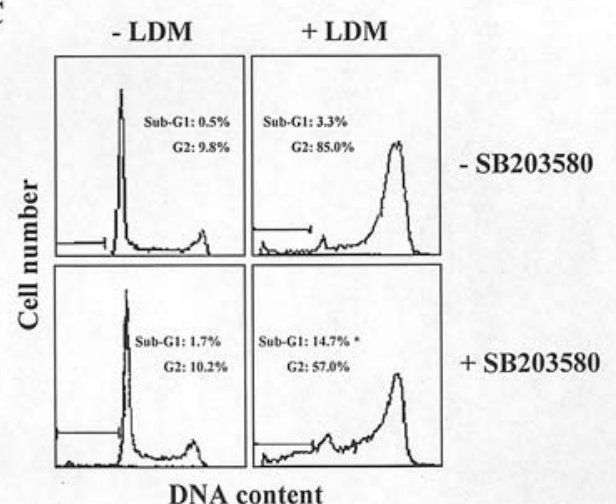

Figure 4. Activation of p38 MAPK is partially involved in LDM-induced G2 arrest. (A) Cells were treated with $0.1 \mathrm{nM}$ LDM for the indicated time, and then the phospho-p38 MAPK expression was assessed by Western blotting using phospho-specific antibody. (B) Cells were treated with $0.1 \mathrm{nM}$ LDM in the presence or absence of $20 \mu \mathrm{M} \mathrm{SB} 203580$ for $48 \mathrm{~h}$. The p38 MAPK activity was assessed by Western blotting using phospho-ATF2 (the p38 substrate) antibody. (C) Cells were treated with $0.1 \mathrm{nM}$ LDM in the presence or absence of $20 \mu \mathrm{M} \mathrm{SB} 203580$ for $48 \mathrm{~h}$, and then analyzed by flow cytometry after PI staining. All data are representative of three independent experiments. " $\mathrm{p}<0.05$, compared with LDM treatment alone. treatment, whereas in cells synchronized at $\mathrm{M}$ phase, cyclin B1 was mainly localized in the nucleus (Fig. 3). These data suggest that cytoplasmic localization of cyclin B1 also contributes to LDM-induced G2 arrest.

Activation of p38 MAPK is involved in LDM-induced G2 arrest. Recent reports suggested that p38 MAPK signaling pathway might mediate G2 cell cycle arrest (10-12). Therefore, we tested the effect of LDM on p38 MAPK using phosphospecific antibody (its activation form). As shown in Fig. 4A, exposure of HT-29 cells to $0.1 \mathrm{nM}$ LDM resulted in significant and sustained activation of p38 MAPK. To further study whether p38 MAPK activation is associated with LDMinduced G2 arrest, we used SB203580, a specific p38 MAPK inhibitor, to inhibit the p38 activation. As shown in Fig. 4B, phosphorylation of the transcription factor ATF2, a downstream target of p38 MAPK, was markedly increased after LDM treatment, which supported the result that p38 MAPK pathway was activated in LDM-treated HT-29 cells. However, in the presence of SB203580, LDM-induced ATF2 phosphorylation was obviously decreased compared with LDM treatment alone (Fig. 4B), suggesting that activation of p38 MAPK induced by LDM was inhibited by SB203580.

Subsequently, to evaluate the contribution of the p38 MAPK activation to LDM-induced G2 arrest, we investigated the cell cycle alteration of LDM-treated HT-29 cells in the presence and absence of SB203580 by flow cytometry. As shown in Fig. 4C, pretreatment with SB203580 for $1 \mathrm{~h}$ attenuated the LDM-induced G2 population accumulation (decrease from $85 \%$ to $57 \%$ ), but could not completely abolish the G2 arrest. Taken together, these results suggest that p38 MAPK pathway partially mediates the LDM-induced G2 arrest.

Inhibition of $p 38$ MAPK decreases the phosphorylated level of $C d c 2$. To further investigate the mechanism by which 


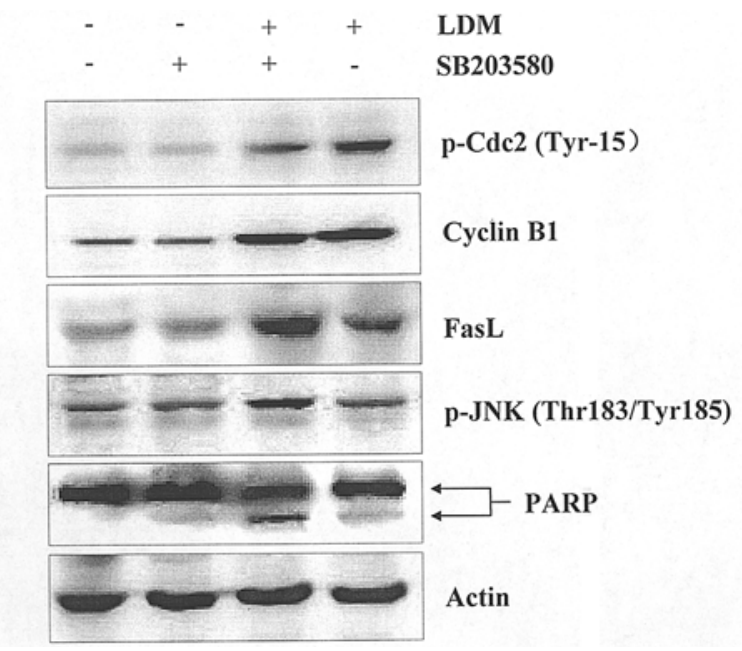

Figure 5. Effects of SB203580 on Cdc2 and JNK phosphorylation, and FasL expression. Cells were treated with $0.1 \mathrm{nM}$ LDM in the presence or absence of $20 \mu \mathrm{M}$ SB203580 for $48 \mathrm{~h}$. Cell lysates were prepared for SDS-PAGE and analyzed by Western blotting as described in 'Materials and methods'. Actin was used as a loading control. Data are representative of three independent experiments.

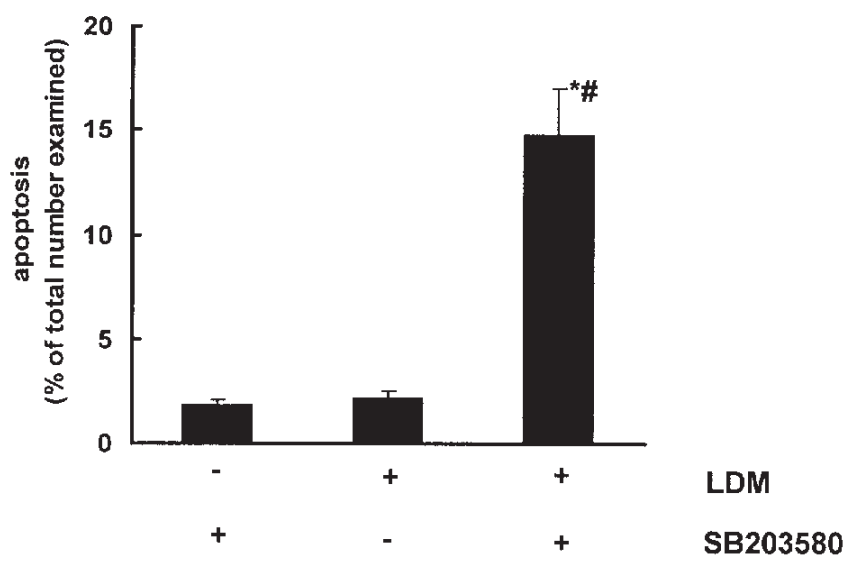

Figure 6. SB203580 potentiates LDM-induced apoptosis. Cells were treated with $0.1 \mathrm{nM} \mathrm{LDM}$ in the presence or absence of $20 \mu \mathrm{M} \mathrm{SB} 203580$ for $48 \mathrm{~h}$, then imaged using an inverted fluorescent microscope as described in 'Materials and methods'. The percentage of apoptotic death cells was calculated from the number of cells with apoptotic nuclear morphology divided by the total number of cells examined. Data are representative of three independent experiments. Original magnification, $x 200 .{ }^{*} \mathrm{p}<0.05$, compared with SB treatment alone. ${ }^{*} \mathrm{p}<0.05$, compared with LDM treatment alone.

inhibition of p38 MAPK can attenuate LDM-induced G2 arrest, we tested the expression of cyclin B1 and the phosphorylated level of Cdc2 in the presence and absence of SB203580. We found that pretreatment with SB203580 for $1 \mathrm{~h}$ decreased the phosphorylated levels of Cdc2 but had no influence on cyclin B1 expression compared with LDM treatment alone (Fig. 5). These data suggest that inhibition of p38 MAPK can protect $\mathrm{Cdc} 2$ from inactivation by LDM, thereby reducing the LDMinduced G2 arrest.

Inhibition of p38 MAPK potentiates cell death induced by $L D M$. Unexpectedly, we found that pretreatment with
SB203580 for $1 \mathrm{~h}$ not only reduced the LDM-induced G2 arrest, but also increased the sub-G1 population compared with LDM treatment alone (Fig. $4 \mathrm{C}, \mathrm{p}<0.05$ ), implying that cell death induced by LDM was increased after inhibition of p38 MAPK. This result was confirmed by Hoechst 33342 staining (Fig. 6). In comparison with LDM treatment alone, the percentage of cells with apoptotic nuclear morphology was significantly increased in SB203580 and LDM cotreated cells (Fig. 6B, p<0.05). Moreover, the cleavage fragment of PARP was also increased in LDM and SB203580 co-treated cells (Fig. 5), supporting the result that inhibition of p38 MAPK can enhance LDM-induced cell death in HT-29 cells.

Inhibition of p38 MAPK increases the levels of phosphorylated JNK and FasL. Next, we investigated the mechanism by which pretreatment with SB203580 potentiated the LDMinduced cell death. In the apoptosis-related molecules, we found that only the expression of FasL was increased in SB203580 and LDM co-treated cells compared with LDM treatment alone (Fig. 5), whereas the expression of Bcl-2 and Bax was unchanged (data not shown), indicating that inhibition of p38 MAPK might activate the FasL/Fas apoptosis pathway in LDM-treated cells, thereby leading to an enhanced susceptibility of cells to LDM-induced cell death. Surprisingly, we found that although the activation (phosphorylated) of JNK was unchanged in LDM-treated HT-29 cells, the combination of LDM and SB203580 markedly increased the phosphorylated level of JNK compared with LDM single treatment (Fig. 5), suggesting JNK was activated in LDM and SB203590 co-treated cells.

Cells at G1 phase are sensitive to LDM. We also investigated whether the sensitivity of HT-29 cells to LDM is cell cycle dependent. Synchronized cells (Fig. 7A) were released by replacing medium containing $10 \%$ serum at different cell cycle phases, and then treating with $0.1 \mathrm{nM}$ LDM for $48 \mathrm{~h}$. Cells were collected and analyzed by flow cytometry. Results showed that cells synchronized at G1 phase are more sensitive to LDM compared with cells synchronized at $\mathrm{S}$ and G2/M (Fig. 7B), and the sub-G1 populations are $22.4 \%, 10.8 \%$ and $3.9 \%$, respectively. These data imply that LDM may be a cell cycle-dependent DNA damaging agent.

\section{Discussion}

LDM is currently being evaluated in clinical trials as a potential anticancer agent in P.R. China. Our previous study showed that LDM-induced different cell cycle arrests were dependent on cell lines (22). In this study, we focused on the mechanisms and signaling pathways of LDM-induced cell cycle arrest in HT-29 cells. Using PI staining, the data showed that LDM induced cell cycle arrest in G2/M phase. We further confirmed that LDM principally induced G2 phase arrest by MPM-2/PI bivariate flow cytometry. The data suggest that LDM induces distinctly G2 arrest in p53 mutant human colon cancer cells.

Chk1 and Chk2 are key elements of the DNA damageinduced G2 checkpoint. Chk1 and Chk2 are activated in response to DNA damage and phosphorylate $\mathrm{Cdc} 25 \mathrm{C}$ on serine-216. Phosphorylated Cdc25C is then complexed with 
A

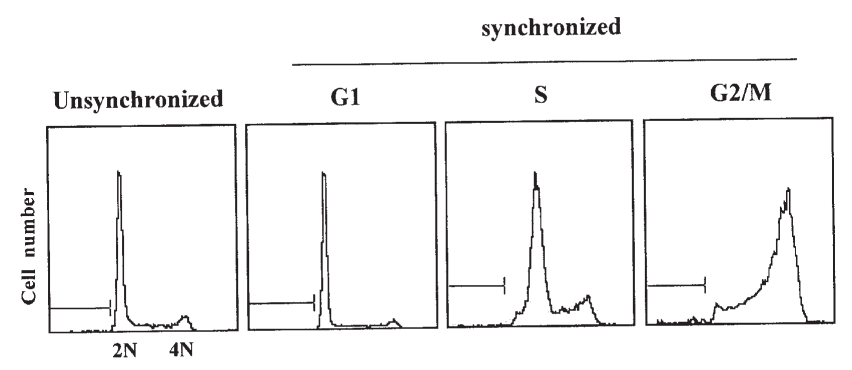

B

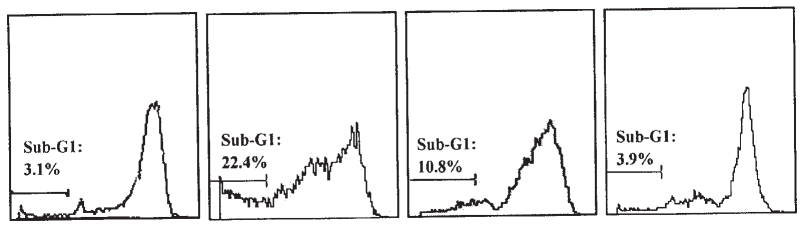

Figure 7. Cells at G1 phase are more sensitive to LDM. Cells were synchronized as described in 'Materials and methods'. Then cells were released by replacing medium containing $10 \%$ serum with $0.1 \mathrm{nM}$ LDM for 48 h. Cells were collected and analyzed by flow cytometry after PI staining. Unsynchronized cells were served as a control and the sub-G1 population represents the dead cells. Data are representative of three independent experiments.

14-3-3 protein, and inhibits the ability of Cdc25C to activate $\mathrm{Cdc} 2 /$ cyclin B1, resulting in G2 cell cycle arrest (7). We found that phosphorylation of Chk1 and Chk2 was increased after LDM treatment, which was consistent with our previous study (22). Because these phosphorylated levels represented their activation forms, these data indicated that both Chk1 and Chk2 pathways were activated by LDM. Furthermore, both phosphorylated $\mathrm{Cdc} 25 \mathrm{C}$ and phosphorylated $\mathrm{Cdc} 2$ were also increased, which confirmed activation of Chk1 and Chk2. Regarding the increases of cyclin $\mathrm{B} 1$ and $\mathrm{Cdc} 2$ proteins, these changes may be related with the increase in proportion of cells at G2 phase, and are not associated with kinase activity, which is consistent with a previous report (29). These data indicated that Chk1 and Chk2 negatively regulated Cdc2 kinase by phosphorylating on tyrosine-15 in LDM-treated cells. Thus, we propose that DNA damage induced by LDM activates Chk1 and Chk2 kinases guarding against mitotic entry from $\mathrm{G} 2$ phase.

The biological function of $\mathrm{Cdc} 2 /$ cyclin $\mathrm{B} 1$ is regulated by changes in subcellular localization. Some reports have demonstrated that G2 cell cycle arrest is further assured by stabilizing the cytoplasmic localization of cyclin B 1 $(8,9,30,31)$. Fig. 3 shows that cyclin B1 accumulated in the cytoplasm in LDM-treated cells, supporting that cytoplasmic localization of cyclin B1 could contribute to G2 arrest induced by LDM in HT-29 cells. So far, we cannot determine how LDM causes cytoplasmic localization of cyclin B1. It is necessary in further studies to confirm whether LDM promotes nuclear export of cyclin B1 or inhibits its import into the nucleus.

It has been reported that p38 MAPK is involved in G2 checkpoint pathway. Activation of p38 MAPK is required for the $\gamma$-radiation-induced G2 arrest (32) and p38 MAPK has a crucial role in the initiation of $\mathrm{G} 2 / \mathrm{M}$ checkpoint in response to UV radiation (10). Consistent with this notion, our results showed that p38 MAPK was activated in LDM-treated cells. Furthermore, we found that inhibition of p38 MAPK by SB203580 partially reduced the phosphorylated level of Cdc2 compared with LDM alone. It is well known that reduced activity of Cdc2 is essential for the cell cycle arrest at G2 phase (33). Thus, protection of Cdc2 inactivation by LDM may be the reason why inhibition of p38 MAPK can lead to attenuation of the G2 arrest, indicating that p38 MAPK pathway was associated with LDM-induced G2 arrest.

Cell cycle arrest may provide the time for damaged-DNA repairing, thereby increasing the chance of cell survival. This suggests that abolition of cell cycle arrest may enhance the sensitivity of cells to DNA damaging agents. The previous study indicated that cell cycle checkpoint abrogator UCN-01 enhances the cytotoxicity of DNA damaging agents in p53deficient cells, which is consistent with this notion (34). In this study, we found that inhibition of p38 MAPK attenuated LDM-induced G2 arrest, and potentiated LDM-induced cell death. Our data raises the possibility that combination of LDM with p38 MAPK inhibitor may be a new strategy for colon cancer therapy.

Further study demonstrated that the increase in cell death might be related with increasing FasL expression. In addition, the JNK was activated in LDM and SB203580 co-treated cells (Fig. 5). A recent report has demonstrated that FasL is a target in the JNK/c-jun signaling pathway that induces apoptosis (35). We suppose that activation of JNK and subsequent increase of FasL by co-treatment with LDM and SB203580 are necessary for HT-29 cells undergoing apoptosis. Our data also indicated that LDM-induced cell death was dependent on cell cycle progression. Fig. 7 shows that the apoptotic sub-G1 peak was higher in cells synchronized at $\mathrm{G} 1$ phase than cells synchronized at $\mathrm{S}$ and $\mathrm{G} 2 / \mathrm{M}$ phases. These results may imply that the sensitivity of cells to LDM is correlated with DNA synthesis.

In summary, our findings suggest that LDM induces distinctly G2 arrest in p53 mutant human colon carcinoma HT-29 cells. Moreover, this is the first report that p38 MAPK is associated with LDM-induced cell cycle arrest and cell death. Although there might be other mechanisms and signaling pathways to regulate the activity of LDM, the present study identifies at least a possibility that p38 MAPK may be a target for sensitizing colon cancer cells to LDM.

\section{Acknowledgments}

This study was supported by grants from NSFC (no. 30025043 , 30472107), the National 973 Program (no. 2002CB513108) and NFCR (USA).

\section{References}

1. Caporali S, Falcinelli S, Starace G, Russo MT, Bonmassar E, Jiricny J and D'Atri S: DNA damage induced by temozolomide signals to both ATM and ATR: role of the mismatch repair system. Mol Pharmacol 66: 478-491, 2004

2. Norbury CJ and Zhivotovsky B: DNA damage-induced apoptosis. Oncogene 23: 2797-2808, 2004.

3. Zhou BB and Elledge SJ: The DNA damage response: putting checkpoints in perspective. Nature 408: 43343-43349, 2000 
4. Melchionna R, Chen XB, Blasina A and McGowan CH: Threonine 68 is required for radiation-induced phosphorylation and activation of Cds1. Nat Cell Biol 2: 762-765, 2000.

5. Zhao H and Piwnica-Worms H: ATR-mediated checkpoint pathways regulate phosphorylation and activation of human chk1. Mol Cell Biol 21: 4129-4139, 2001.

6. Liu Q, Guntuku S, Cui XS, Matsuoka S, Cortez D, Tamai K, Luo G, Carattini-Rivera S, DeMayo F, Bradley A, Donehower LA and Elledge SJ: Chk1 is an essential kinase that is regulated by Atr and required for the G2/M DNA damage checkpoint. Genes Dev 14: 1448-1459, 2000.

7. Abraham RT: Cell cycle checkpoint signaling through the ATM and ATR kinases. Genes Dev 17: 2177-2196, 2001.

8. Toyoshima F, Moriguchi T, Wada A, Fukuda M and Nishida E: Nuclear export of cyclin B1 and its possible role in the DNA damage-induced G2 checkpoint. EMBO J 17: 2728-2735, 1998.

9. Jin P, Hardy S and Morgan DO: Nuclear localization of cyclin B1 controls mitotic entry after DNA damage. J Cell Biol 141: 875-885, 1998

10. Bulavin DV, Higashimoto Y, Popoff IJ, Gaarde WA, Basrur V, Potapova O, Appella E and Fornace AJ Jr: Initiation of a G2/M checkpoint after ultraviolet radiation requires p38 kinase. Nature 411: 102-107, 2001.

11. Dmitrieva NI, Bulavin DV, Fornace AJ Jr and Burg MB: Rapid activation of $\mathrm{G} 2 / \mathrm{M}$ checkpoint after hypertonic stress in renal inner medullary epithelial (IME) cells is protective and requires p38 kinase. Proc Natl Acad Sci USA 99: 184-189, 2002.

12. Hsu YL, Kuo PL, Lin LT and Lin CC: Asiatic acid, a triterpene, induces apoptosis and cell cycle arrest through activation of extracellular signal-regulated kinase and p38 mitogen-activated protein kinase pathways in human breast cancer cells. J Pharmacol Exp Ther 313: 333-344, 2005.

13. Tang D, Wu D, Hirao A, Lahti JM, Liu L, Mazza B, Kidd VJ, Mak TW and Ingram AJ: ERK activation mediates cell cycle arrest and apoptosis after DNA damage independently of p53. J Biol Chem 277: 12710-12717, 2002.

14. Goulet AC, Chigbrodw M, Frisk P and Nelson MA: Selenomethionine induces sustained ERK phosphorylation leading to cell-cycle arrest in human colon cancer cells. Carcinogenesis 26: 109-117, 2005.

15. Kuntzen C, Sonuc N, De Toni EN, Opelz C, Mucha SR, Gerbes AL and Eichhorst ST: Inhibition of c-Jun-N-terminalkinase sensitizes tumor cells to CD95-induced apoptosis and induces G2/M cell cycle arrest. Cancer Res 65: 6780-6788, 2005.

16. Mingo-Sion AM, Marietta PM, Koller E, Wolf DM and Van Den Berg CL: Inhibition of JNK reduces G2/M transit independent of p53, leading to endoreduplication, decreased proliferation, and apoptosis in breast cancer cells. Oncogene 23: 596-604, 2004.

17. Lu J, Quearry B and Harada H: p38-MAP kinase activation followed by BIM induction is essential for glucocorticoidinduced apoptosis in lymphoblastic leukemia cells. FEBS Lett 580: 3539-3544, 2006.

18. Sun Y and Sinicrope FA: Selective inhibitors of MEK1/ERK44/42 and p38 mitogen-activated protein kinases potentiate apoptosis induction by sulindac sulfide in human colon carcinoma cells. Mol Cancer Ther 4: 51-59, 2005.

19. Zhang R, Otani T, Minami Y, Yamada Y and Marunaka T: A new macromolecular antitumor antibiotic, C-1027. I. Discovery, taxonomy of producing organism, fermentation and biological activity. J Antibiot 41: 1575-1579, 1988.
20. Xu YJ, Zhen YS and Goldberg IH: C1027 chromophore, a potent new enediyne antitumor antibiotic, induces sequencespecific double-strand DNA cleavage: Biochemistry 33: 5947-5954, 1994.

21. McHugh MM, Gawron LS, Matsui S and Beerman TA: The antitumor enediyne C-1027 alters cell cycle progression and induces chromosomal aberrations and telomere dysfunction. Cancer Res 65: 5344-5351, 2005.

22. Liu X, He H, Feng Y, Zhang M, Ren K and Shao R: Difference of cell cycle arrests induced by lidamycin in human breast cancer cells. Anticancer Drugs 17: 173-179, 2006.

23. Chen ZY, Shie JL and Tseng CC: Gut-enriched Kruppel-like factor represses ornithine decarboxylase gene expression and functions as checkpoint regulator in colonic cancer cells. J Biol Chem 277: 46831-46839, 2002.

24. Takahashi N, Li W, Banerjee D, Guan Y, Wada-Takahashi Y, Brennan MF, Chou TC, Scotto KW and Bertino JR: Sequencedependent synergistic cytotoxicity of ecteinascidin-743 and paclitaxel in human breast cancer cell lines in vitro and in vivo. Cancer Res 62: 6909-6915, 2002.

25. Shirin H, Pinto JT, Kawabata Y, Soh JW, Delohery T, Moss SF, Murty V, Rivlin RS, Holt PR and Weinstein IB: Antiproliferative effects of S-allylmercaptocysteine on colon cancer cells when tested alone or in combination with sulindac sulfide. Cancer Res 61: 725-731, 2001.

26. Davis FM, Tsao TY, Fowler SK and Rao PN: Monoclonal antibodies to mitotic cells. Proc Natl Acad Sci USA 80: 2926-2930, 1983.

27. Bunz F, Dutriaux A, Lengauer C, Waldman T, Zhou S, Brown JP, Sedivy JM, Kinzler KW and Vogelstein B: Requirement for p53 and p21 to sustain G2 arrest after DNA damage. Science 282: 1497-1501, 1998

28. Takagaki N, Sowa Y, Oki T, Nakanishi R, Yogosawa S and Sakai T: Apigenin induces cell cycle arrest and p21/WAF1 expression in a p53-independent pathway. Int J Oncol 26: 185-189, 2005.

29. Ding Z, Parchment RE, LoRusso PM, Zhou JY, Li J, Lawrence TS, Sun Y and Wu GS: The investigational new drug XK469 induces G(2)-M cell cycle arrest by p53-dependent and -independent pathways. Clin Cancer Res 7: 3336-3342, 2001.

30. Hagting A, Karlsson C, Clute P, Jackman M and Pines J: MPF localization is controlled by nuclear export. EMBO J 17: 4127-4138, 1998.

31. Morita E, Tada K, Chisaka H, Asao H, Sato H, Yaegashi N and Sugamura K: Human parvovirus B19 induces cell cycle arrest at $\mathrm{G}(2)$ phase with accumulation of mitotic cyclins. J Virol 75 : 7555-7563, 2001.

32. Wang X, McGowan CH, Zhao M, He L, Downey JS, Fearns C, Wang Y, Huang $S$ and Han J: Involvement of the MKK6p38gamma cascade in gamma- radiation- induced cell cycle arrest. Mol Cell Biol 20: 4543-4552, 2000.

33. Morgan DO: Principles of CDK regulation. Nature 374: 131-134, 1995.

34. Shao RG, Cao CX and Pommier Y: Abrogation of Chk1mediated S/G2 checkpoint by UCN-01 enhances ara-C-induced cytotoxicity in human colon cancer cells. Acta Pharmacol Sin 25: 756-762, 2004.

35. Mansouri A, Ridgway LD, Korapati AL, Zhang Q, Tian L, Wang Y, Siddik ZH, Mills GB and Claret FX: Sustained activation of JNK/p38 MAPK pathways in response to cisplatin leads to Fas ligand induction and cell death in ovarian carcinoma cells. J Biol Chem 278: 19245-19256, 2003. 